\title{
Time-frequency Characteristics of Micro-seismic Signals Before and after Rock Burst
}

\author{
Xinxin Wang \\ line 2: Shandong University of Science \\ and Technology \\ line 3: College of Mining and Safety \\ Engineering \\ line 4: Qingdao, China \\ line 5: $1037291451 @ q q . c o m$
}

\author{
Shijian Yu \\ line 2: Shandong University of Science \\ and Technology \\ line 3: College of Mining and Safety \\ Engineering \\ line 4: Qingdao, China \\ line 5: 439713892@qq.com
}

\author{
Dawei Yin \\ line 2: Shandong University of Science \\ and Technology \\ line 3: College of Mining and Safety \\ Engineering, State Key Laboratory of \\ Mine Disaster Prevention and Control \\ line 4: Qingdao, China \\ line 5:949251142@qq.com
}

\begin{abstract}
In this paper, the time-frequency characteristics of micro-seismic signals before and after the rock burst occurred in the $\mathbf{1 3 0 0}$ working face of a coal mine in eastern China were analyzed. The results show that before rock burst occurrence, the amplitude was small with gentle vibration fluctuations. And the spectrum distribution was concentrated in the high frequency band, belonging to the random vibration signal. However, the micro-seismic signal appeared obvious change at one hour before rock burst occurrence. The amplitude was increased, with obvious vibration fluctuations, and the frequency spectrum distribution moved to the low frequency band. When rock burst occurred, the amplitude increased by more than ten times, and the frequency spectrum was mainly concentrated in the low frequency band of $0 \sim 80 \mathrm{~Hz}$ At the end of the rock burst, the amplitude was reduced. And the low frequency component was reduced, and the spectrum distribution was mainly concentrated in the high frequency band of 130 350 Hz. It can be concluded that rock burst had obvious precursory features before it occurred, therefore the precursory information can be used to predict rock burst occurrence.
\end{abstract}

Keywords - rock burst, micro-seismic signal, time-frequency characteristics, precursory information

\section{INTRODUCTION}

With the increase of mining depth and mining under complex geological conditions, the rock burst hazard has become increasingly serious, which has become one of the most important factors affecting the safe mining of coal mines. Therefore, the forecasting and prevention of rock burst have become an urgent problem to be solved at the present stage. There will be some geological changes before rock burst occurrence. If we study these changes, we can effectively predict the occurrence of ground pressure disasters. However, due to the complexity of the occurrence conditions of rock burst, the limitation of on-site monitoring methods and the insufficient utilization of monitoring information, there is a certain error in the prediction of rock burst. Micro-seismic monitoring technology can be used to monitor the micro-seismic waves of rock mass stereoscopically in real-time, continuously, and full range. With its own advantages, it has become an effective monitoring tool for forecasting the dynamic disasters in coal mines ${ }^{[1-2]}$.

Therefore, a large number of domestic and foreign scholars use micro-seismic technology to study the characteristics before and after the occurrence of rock burst. Yuan Ruifu analyzed the micro-seismic signals before and after coal pillar rock burst of Pingmei No. 11 coal mine from the perspective of time series characteristics and spatial distribution fractal dimension values ${ }^{[3]}$. Zhao Yixin studied the precursory information of micro-seismic events in rock mass dynamic disasters with hard roof from the relationship between micro-seismic energy level and frequency ${ }^{[4]}$. Li Zhihua used the experiments to refine the features of the precursory micro-seismic signal of fault slip and instability ${ }^{[5]}$. Wei Sijiang analyzed the main shock waveforms of the impact rock mass or large energy microseismic events in the Yimei coal mine area ${ }^{[6]}$. Lu Jinuo used the ARIMA model and the threshold autoregressive model to analyze the high-energy change events, and obtained the activity trend of the microseisms changing with time ${ }^{[7]}$. N.N. Mernikov et al. studied the strain precursors of microseismic events in the Kola Peninsula ${ }^{[8]}$. O.A. Hachay et al. used seismological catalogue data to study rock burst precursors ${ }^{[9]}$.

These studies have analyzed the precursor information of micro-seismic signals from various angles. In this paper, we further analyzed the time-frequency characteristics of this information. It was analyzed of a large number of microseismic signals before and after the rock burst occurred in the 1300 working face of a coal mine. The time-frequency information was obtained by Fourier transform with MATLAB software. In accordance with the order of precedence, the time-frequency diagrams at different time points on the same frequency band were compared. And the different characteristics of micro-seismic signals in frequency before and after rock burst occurrence were studied to extract precursory information and to predict rock burst occurrence ${ }^{[10-11]}$.

\section{ENGINEERING BACKGROUND}

\section{A. 1300 Working face}

The 1300 working face is the first coal mining face. The buried depth is $859 \sim 887 \mathrm{~m}$, and the strike length is $1255 \mathrm{~m}$. The prone length is $100 \mathrm{~m}$, and the coal seam inclination is $3 \sim 6^{\circ}$. The 1300 working face is located in the west of the first mining district. 1300 working face drainage lane in the east. In the west, there are the Balizhuang fault and the 
Bashizhuang branch four faults. In the east, there are 1300 working face drainage tunnels. In the north, there is track of the first mining district. In the south, there are the Balizhuang branch four faults. The location of the coal mine is shown in Fig. 1 (a). According to the physical and mechanical properties test and the impact tendency report, the $3 \#$ coal seam has a weak impact tendency, so the coal mine has the possibility of impact damage ${ }^{[12]}$.

\section{B. Micro-seismic Monitoring System Layouts}

The coal mine uses the ARAMIS M/E micro-seismic monitoring system, which can monitor the entire range of micro-seismic events in real -time. There are several vibration pickups in the coal mine, some of which are distributed as shown in Fig 1(b). Among them, the measurement point installation is as follows: 1300 working face is equipped with 10 stations per channel. As the working face progresses, the station should be disassembled timely and moved forward to make it as close to the area to be measured as possible to ensure that the monitoring range is not less than $200 \mathrm{~m}$. Two measuring points are arranged for each station. The installation depth of the borehole stress is $7 \mathrm{~m}$ and $14 \mathrm{~m}$, with an interval of $1 \mathrm{~m}$.

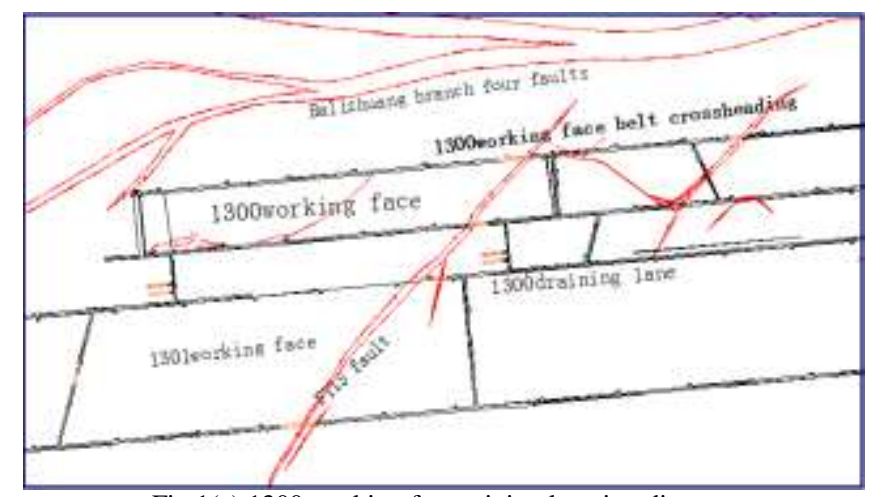

Fig.1(a) 1300 working face mining location diagram

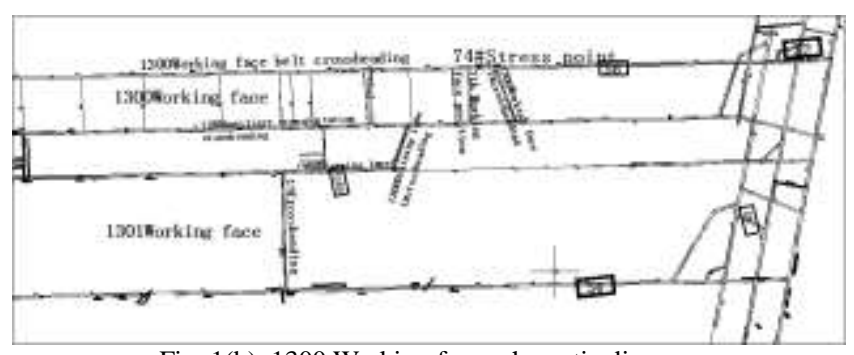

Fig. 1(b) 1300 Working face schematic diagram

\section{Rock Burst Condition}

In order to analyze the time-frequency characteristics of the mine during rock burst occurrence, taking the microseismic data of 1300 working face in July 2015 as an example, a significant rock burst event received on July 7 was studied. The time for the occurrence of rock burst was $2: 54 \mathrm{pm}$ on the 7th. On the west of original 1300 drainage tunnels of the $3 \#$ contact lane (at a distance of $263 \mathrm{~m}$ from the 1300 working face of the same day), it had a rock burst event with an energy of $2.1 \times 10^{4} \mathrm{~J}$ and the magnitude reached level 1.3. At the same time, it was monitored that KJ550 had a yellow warning at the 1300 working face $74 \#$ stress point(distance from working face $67.4 \mathrm{~m}$ ), and the pressure values at other measurement points also increased in different degrees. The laneway of 1301 belt crossheading was no obvious change from the return air entrance to the 1301 working face $2 \#$ crossheading. The Fig1.shows rock burst of 1300 working face, in which the black circle is the location of the rock burst.

\section{TIME-FREQUENCY ANALYSIS OF MICRO-SEISMIC}

The coal mine uses the ARAMIS M/E micro-seismic monitoring system. Since the vibration pick-ups are different from the source, the detected signal intensity is also different. For this reason, it was the focus of timefrequency analysis by selecting a vibration pick-up that can detect noticeable signal changes. In this article, taking the S2 vibration pick-up as an example for analysis, the S2 vibration pick-up is installed in the return air contact laneway of 1300 the tape. It is about $820 \mathrm{~m}$ away from the rock burst occurred.

In order to clearly understand the foreshock conditions of the rock burst, the following two micro-seismic conditions before rock burst occurrence were analyzed and studied. Fig. 2 shows the time-frequency diagram of two hours before the rock burst occurrence. Fig. 2 shows the time-frequency diagram of one hour before rock burst occurrence.

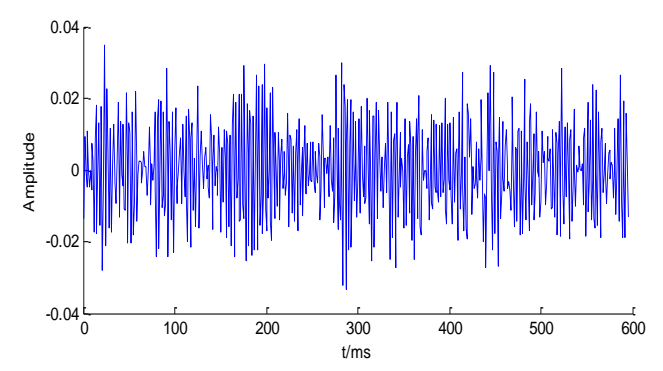

(a) Time domain figure

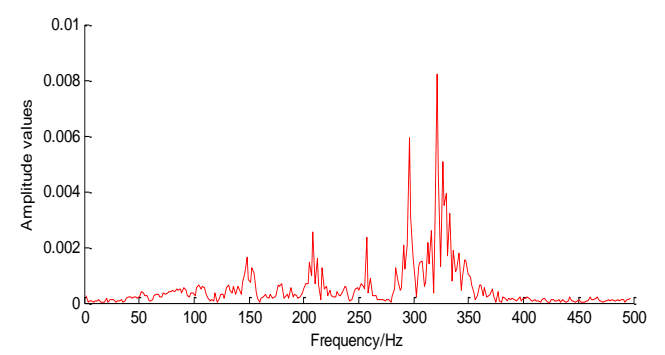

(b) Spectrogram

Fig.2 Time-frequency diagram of micro-seismic signals at 0:56:54 on the $7^{\text {th }}$

It can be seen from the Fig. 2 that two hours before the rock burst occurred. In the time domain, the amplitude was very small with gentle the vibration fluctuation, belonging to the random vibration signal. In the frequency spectrum, there were few the low-frequency signals. The highfrequency signals occupied the mainstream, which was mainly concentrated in $200 \sim 350 \mathrm{~Hz}$. It can be seen from Fig. 3 that one hour before the rock burst occurred. The vibration fluctuations began to become apparent, with the amplitude increasing. The low-frequency signal increased, and the frequency was mainly concentrated in $100 \sim 350 \mathrm{~Hz}$. It means that the rock burst precursor had already appeared at one hour before the occurrence of rock burst. At the same time comparing Fig. 2 with Fig. 3, it can be seen that the closer to the moment of rock burst, the larger the amplitude was, and the more obvious the waveform vibration and low- 
frequency components appear. In the shock one hour before the rock burst occurrence, the frequency of low-frequency components were significantly higher than before two hours. Therefore, when the vibration signal continues to increase in amplitude and the low-frequency component continues to increase, it can be determined that the risk of high-energy vibration will increase.

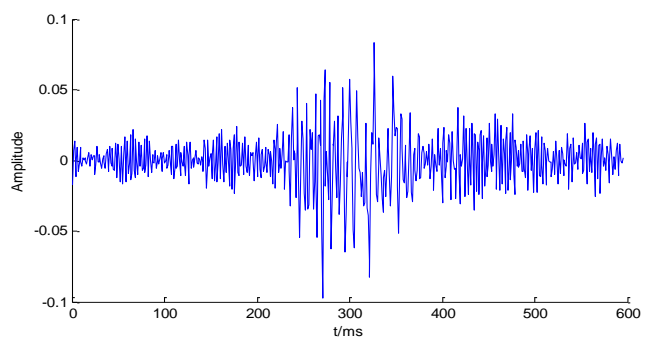

(a) Time-domain figure

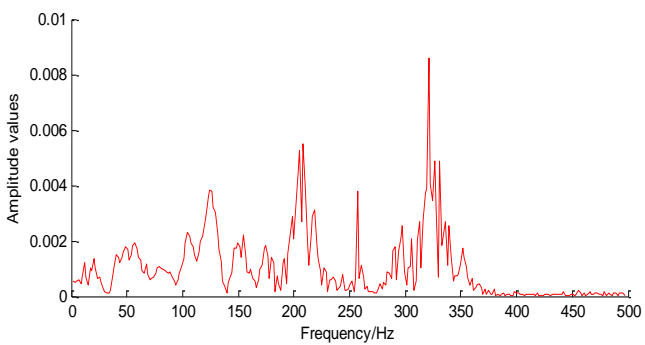

(b) Spectrogram

Fig. 3 Time-frequency diagram of micro-seismic signal at 1:51:09 on the 7 th

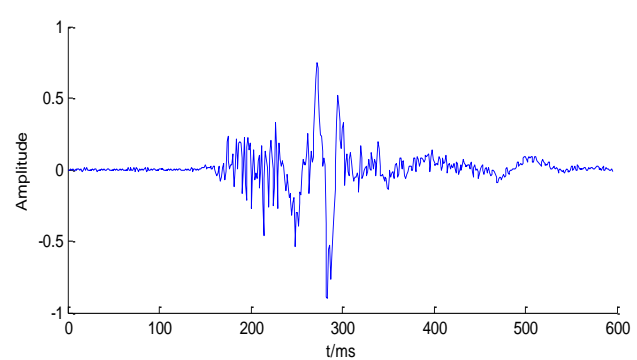

(a)Time-domain figure

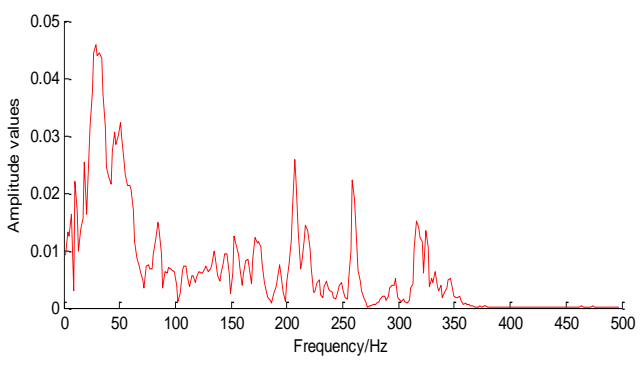

(b) Spectrogram

Fig. 4 Time-frequency diagram of rock burst at 2:54:59 on the 7th

Fig. 4 shows the time-frequency diagram of the rock burst occurred. It can be seen from the figure that there is an obvious low-frequency signal. The signals lasted for about $0.2 \mathrm{~s}$ and the amplitude was dozens of times larger than before the rock burst occurred. The high amplitude duration was very short and the tail wave was very obvious. In the frequency spectrum, the amplitude value was larger. And compared with the foreshocks of the rock burst occurred, it can be clearly seen that the amplitude value was several times larger. The frequency was mainly concentrated in the range of $0 \sim 80 \mathrm{~Hz}$ and reached the maximum at $30 \mathrm{~Hz}$. The low-frequency signal occupied a major part, especially the composition below $60 \mathrm{~Hz}$. This was rare in foreshocks and other vibrations.
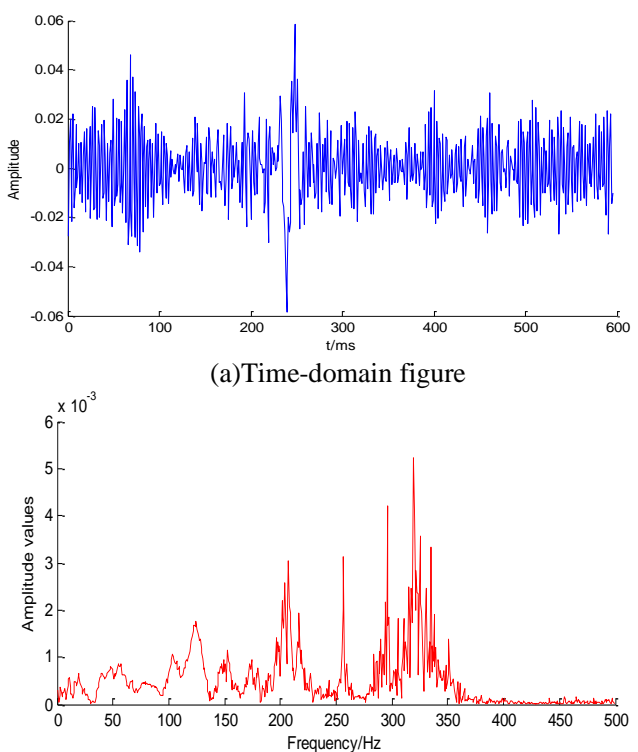

(b) Spectrogram

Fig. 5 Time-frequency diagram of micro-seismic signals at 3:05:56 on the $7^{\text {th }}$

Fig. 5 shows the time-frequency diagrams collected by the micro-seismic monitoring system after the 9 minutes rock burst occurred. As can be seen from the figure, the waveform vibration became gentle and the amplitude was less than a few times that of the rock burst occurred. In the frequency spectrum, the amplitude value was also greatly reduced, with less low frequency components. The main frequency of micro-seismic signals was high, which was mainly concentrated in $130 \sim 350 \mathrm{~Hz}$. It can be seen that with the passage of time, the waveform vibration became more and more smooth and gradually recovered to the random vibration signal. And the low frequency signal gradually decreased, and the high frequency signal gradually increased. This was contrary to the changing characteristics of the foreshock signal.

Therefore, by analyzing the time-frequency characteristics before and after the occurrence of rock burst, the vibrations in the same frequency band were compared. It can be concluded that the precursor of the rock burst was that the vibration of the micro-seismic signal become obvious. And the amplitude began to increase, and the frequency shifted from the high frequency band to the low frequency band. The time of the foreshock was closer to the main shock, the more low frequency signals were. Thus, a sharp decrease in the frequency of the micro-seismic and a significant increase in the amplitude can be used as a feature of the rock burst precursor.

\section{CONCLUSION}

The time-frequency characteristics of micro-seismic signals before and after the rock burst occurred on the 1300 working face of a mine was analyzed. It shows that there was obvious precursory information before rock burst occurrence, which can be used as one of the means to predict rock burst occurrence.

Two hours before the occurrence of rock burst, the amplitude was small and the fluctuation was stable, which 
belong to the random vibration signal. The frequency spectrum distribution was mainly concentrated in the high frequency range of $200 \sim 350 \mathrm{~Hz}$. However, the rock burst precursor has appeared at one hour before the occurrence of the rock burst. The amplitude was increased, with obvious vibration fluctuations, and the frequency spectrum distribution moved to the low frequency band. The frequency spectrum distribution was mainly concentrated in the high frequency range of $100 \sim 350 \mathrm{~Hz}$. When the rock burst occurred, the amplitude was dozens of times larger than before the rock burst occurred, and the frequency spectrum was mainly concentrated in the low frequency band of $0 \sim 80 \mathrm{~Hz}$. At the end of the rock burst, the amplitude was decreased, and the spectrum distribution was mainly concentrated in the high frequency range of $130-350 \mathrm{~Hz}$.

\section{ACKNOWLEDGMENT}

We are very grateful to the Shandong Coal Mine for providing us with a large number of data records, and thank you reviewers for their valuable comments.

\section{REFERENCES}

[1] Lu Caiping, Dou Linming, Wu Xingrong, et al. Frequency spectrum analysis on micro-seismic monitoring and signal differentiation of rock material[J]. Chinese Journal of Geotechnical Engineering, 2005, 27(7):77277533(in Chinese).

[2] Li Chengwu, Liu Jikun, Wang Cuixia , et al. Spectrum characteristics analysis of micro-seismic signals transmitting between coal bedding[J]. Safety Science, 50 (2012) 761-767.
[3] Yuan Ruifu, Li Huamin, Li Huaizhen. Distribution of micro-seismic signal and discrimination of portentous information of pillar type rock burst [J]. Chinese Journal of Rock Mechanics and Engineering, 2012, 31(1):8085(in Chinese).

[4] Zhao Yixin, Jiang Yaodong, Wang Tao, et al. Features of micro-seismic events and precursors of rock burst in underground coal mining with hard roof[J]. Journal of China Coal Society, 2012, 37(12): 1960-1966(in Chinese).

[5] Li Zhihua, Dou Linming, Lu Caiping. Frequency spectrum analysis on micro- seismic signal of similar simulation test of fault rock burst [J]. Journal of Shandong University of Science and Technology, 2010, 29(4): 51-56(in Chinese).

[6] Wei Sijiang, Yang Yushun. Spectrum Characteristic Analysis of rock Burst Micro-seismic Signal in Yimei Mining Area[J]. Safety in Coal Mines, 2015,46 (4): 181-188(in Chinese).

[7] Lv Jinguo, Pan Li.Microseismic predicting coal bump by time series method[J].Journal of China coal society,2010,35(12):2002-2005(in Chinese).

[8] N. N. Melnikov;A. A. Kozyrev;V. I. Panin. Induced seismicity in largescale mining in the Kola peninsula and monitoring to reveal informative precursors [J]. Induced Seismic Events, 1996, 263-276.

[9] O.A. Hachay; O.Y. Khachay. Precursors of Rock Bursts in Rock Massif Using Seismological Catalogue Data[J], 7th EAGE Saint Petersburg International Conference and Exhibition, 2016.

[10] Lu Caiping ,Dou Linming , LIU Biao ,et .al. Micro-seismic lowfrequency precursor effect of bursting failure of coal and rock[J]. Journal of Applied Geophysics (79)2012, 55-63(in Chinese).

[11] Yang Zuolin, Micro-seismic signal recognition and the law of ground pressure disaster microseism precursor research [D]. Jiangxi University of Science and Technology,2015(in Chinese).

[12] Jiang Fuxing, Liu Yi, Yang Wei Li, et al. Relationship between rock burst and the three zone structure loading model in Yuncheng coal mine [J]. Journal of Mining and Safety Engineering, 2017,34(3):405-410(in Chinese). 